\title{
ANFIS: Adaptive Neuro-Fuzzy Inference System- A Survey
}

\author{
Navneet Walia \\ Department of Electronics \& \\ Communication, CT Institute of \\ Technology \& Research, \\ Jalandhar, India
}

\author{
Harsukhpreet Singh \\ Department of Electronics \& \\ Communication, CT Institute of \\ Technology \& Research, \\ Jalandhar, India
}

\author{
Anurag Sharma \\ Department of Electronics \& \\ Communication, CT Institute of \\ Technology \& Research, \\ Jalandhar, India
}

\begin{abstract}
In this paper, we presented the architecture and basic learning process underlying ANFIS (adaptive-network-based fuzzy inference system) which is a fuzzy inference system implemented in the framework of adaptive networks. Soft computing approaches including artificial neural networks and fuzzy inference have been used widely to model expert behavior. Using given input/output data values, the proposed ANFIS can construct mapping based on both human knowledge (in the form of fuzzy if-then rules) and hybrid learning algorithm. In modeling and simulation, the ANFIS strategy is employed to model nonlinear functions, to control one of the most important parameters of the induction machine and predict a chaotic time series, all yielding more effective, faster response or settling times.
\end{abstract}

\section{General Terms}

Adaptive Neuro-Fuzzy Inference System, Fuzzy Rules

\section{Keywords}

ANFIS, Fuzzy Logic, Takagi-Sugeno (T-S) Model, Learning Algorithm

\section{INTRODUCTION}

Control of nonlinear systems based on conventional mathematical tools is a difficult problem because no systematic tools are available to deal with ill-defined and uncertain systems. By contrast, a fuzzy inference system employing fuzzy if-then rules can model the qualitative aspects of human knowledge and reasoning processes but lacks standard design procedure to employ precise quantitative analyzes. Neural networks work by detecting patterns in data, learning from the relationships and adapting to them. This knowledge is then used to predict the outcome for new combinations of data [2]. In particular, the control technique based on fuzzy modeling or fuzzy identification was first systematically introduced by Takagi and Sugeno [1], has found numerous applications in fuzzy control, for medical diagnosis [3], decision-making and solve problems based on data mining [4]. However, there are some basic aspects of this approach which are in need of better understanding. More specifically, the lack of standard design procedure and optimization process to transform human knowledge or experience into rule base and the data base of the fuzzy inference system. It is hard to interpret tuning of membership function so as to minimize output error index and to choose appropriate network's structure.

This paper is concerned with novel architecture called Adaptive Neuro-Fuzzy Inference System (ANFIS), has been widely employed to represent or approximate a nonlinear system. Adaptive systems can be described by constructing a set of fuzzy if-then rules that represent local linear inputoutput relations of the system. In recent years, Takagi-Sugeno (T-S) fuzzy models are playing an important role in dealing with problems concerning a wide class of nonlinear systems. Artificial Neural Network can be used as an alternative means for the knowledge about the engine. ANN is based on binary logic which can store knowledge by learning from recorded data. It has been proven that T-S fuzzy systems with affine terms can smoothly approximate any nonlinear functions to any specified accuracy within any compact set, which provides a theoretical foundation for using T-S fuzzy model to represent complex nonlinear system approximately.

\section{LITERATURE SURVEY}

There are various application areas in which ANN and FL have been successfully implemented whether individually or complementing each other's strengths. A combined neurofuzzy approach has seen enormous preferences recently from researchers working in different domains. A comprehensive study of existing work in assorted areas using soft computing methodologies specifically focusing on neural networks and fuzzy logic can be found in [1]. A computational technique to deal with non-linear and complex problem was discussed by J.R Jang (1993). This study involves fuzzy inference system implemented in the construction of adaptive networks. The proposed ANFIS can generate an input-output mapping based on human knowledge and predetermined input-output data pairs using the hybrid algorithm. The simulation studies for system architecture is utilized to model nonlinear functions, identify nonlinear components online in a control system and predicts a chaotic time series, all yields remarkable results. Further, author compared system with artificial neural networks and preliminary tested [1]. A comprehensive survey of neuro-fuzzy rule generation algorithms for real-time applications is examined by S. Mitra et al. (2000). The proposed algorithms use fuzzy sets and an aid in giving information in a more human comprehensible or natural form and can handle uncertainties at various levels. An extensive investigation shows the qualitative better results can be obtained using rule extraction and rule refinement. Models are the group on the basis of their level of neuro-fuzzy synthesis. . The proposed methodology has an additional benefit in other soft other soft computing tools like genetic algorithms and rough sets. Based on fuzzy inference system, real-life application to medical diagnosis is provided [15]. Usefulness of adaptive neuro-fuzzy system for predicting surface roughness in turning operation is examined by S.S Roy (2005). Various input parameter namely cutting speed, feed rate and depth of cut have been used for encoding the problem. Two different membership functions triangular and bell shaped were adopted during the training phase. This approach compares ANFIS values with experimental data for 
both triangular and bell shaped membership functions. The developed model based on first-order Takagi-Sugeno and Kang for turning operation showed a higher prediction accuracy using bell membership function [11]. A novel approach solving problems for the air conditioning system by means of Mamdani and Sugeno-type fuzzy inference models was discussed by Arshdeep et al. (2012). This approach outlines the basic difference between the Mamdani-type FIS and Sugeno-type FIS. This study suggests choosing an enhanced membership function of the two FIS for the air conditioning system. Based on inference system implementation author concluded from this paper that for air conditioning system Mamdani-type FIS and Sugeno-type FIS performs similarly but by using Sugeno-type FIS model it allows the air conditioning system to operate at its full capacity [9]. Medical diagnosis applications of ANFIS were introduced by Tamer (2012). The proposed approach uses Sugeno-type adaptive-network-based fuzzy inference system (ANFIS) to prognosticate the existence of mycobacterium tuberculosis. . Dataset collected from 503 different patient records which are obtained from a private health clinic. The patient record has 30 different attributes which cover demographical and medical test data. ANFIS model was generated by using 250 records. The proposed model classifies the instances with the exactness of $97 \%$, whereas rough set algorithm does the same classification with an accuracy of $92 \%$. This learning has a contribution on forecasting patients before the medical tests [13]. The inaccuracy of mathematical modeling of the plants usually degrades the performance of the controller, especially for nonlinear and complex control problems. Use of ANFIS controller for controlling non-linear system was explained by A.V Gite (2013). The simulation study suggests that ANFIS is the best controller as compared to conventional PID controller. The proposed technique can be used in the temperature water controller [21]. A medical expert system for diagnosing of tuberculosis was proposed by Navneet et al. (2015). The proposed Medical Expert Solution (MES) system was to assist medical doctors to diagnose symptoms related to a given tropical disease, suggest the likely ailment, and advances possible treatment based on the MES diagnosis [28]. Abu-Rub et al. (2013) presented an application of ANFIS for maximum power delivery to the load based on maximum power point tracking. The proposed ANFIS based MPPT offers an enormously fast dynamic response with high accuracy. Authors projected technique is tested for isolated load conditions. Simulation and experimental approaches are used to validate the proposed scheme [22]. C. Loganathan et al. (2014) had successfully considered a system that uses a fuzzy system to characterize knowledge in an interpretable manner and have the learning ability derived from a RungeKutta learning method (RKLM) to adjust its membership functions and parameters in order to augment the system performance. The dilemma to discovery appropriate membership functions and fuzzy rules are often a tiring process of trial and error. It requires users to recognize the data before training, which is usually difficult to achieve when the database is relatively large. To overcome these problems, the author explained a hybrid of back propagation neural network and RKLM can combine the advantages of two systems [23].

\section{FUZZY LOGIC}

Fuzzy set theory, which was initially introduced by Lofti Zahed in 1965, is a powerful tool to deal with the imprecision characteristics in decision-making problems involving uncertainty and vagueness of real world applications [5]. Fuzzy inference is a process of mapping from a given input to an output dataset using the theory of fuzzy sets. Knowledge is encoded as using a set of explicit linguistic rules, which can be easily understood by people without technical expertise. Fuzzy systems implement nonlinear systems using linguistic variables in a straightforward when adequate knowledge about the system is available. The fuzzy logic module was used as a decision-making tool to resolve any uncertainty in the decision made by the neural networks. Fuzzy Set Theory (FST), a dominant tool used to handle imprecision and uncertainty can be used to deal with the concept of partial true and partial false values aiming attractability, stoutness and low cost solutions for real world challenges. Unlike Boolean logic or classical logic, which assumes that every fact is either entirely true or false, fuzzy logic extends Boolean logic to handle vague and imprecise expressions. Fuzzy set theory offers the ability to express the ambiguity of human thinking and translate expert knowledge into computable numerical data. It can deal with linguistic terms which explain its implementation in solving problems in medicine and supplementary areas of application. A fuzzy system consists of a set of fuzzy IF-THEN rules that describe the input-output mapping relationship of the networks $[8,10]$. Hybrid systems utilize methodologies of soft computing (fuzzy logic, neural computing, genetic computing etc.) provide a perspective method to build fuzzy inference system when information about object system incomplete. Main components of fuzzy logic are fuzzification, which translates crisp (real-valued) inputs into fuzzy values; rule base reasoning, an inference engine that applies a fuzzy reasoning mechanism to obtain a fuzzy output using rules; and defuzzification, which translates this latter output into a crisp value, as shown in "figure 1". The purpose of fuzzification is to map system input values from 0 to 1 via defined input membership functions. In rulebased reasoning, the fuzzy input values membership values are mapped to classify fuzzy output through a table containing if-then rules. Rules are expressed as a logic implication $p \rightarrow q$ where $p$ is called the antecedent of the rule and $q$ is called the consequence of the rule [16]. Defuzzification is a process which produces single system output (crisp) values by using a defuzzification formula and fuzzy output membership outputs. The fuzzy inference system is a popular computing framework based on the concepts of fuzzy set theory, fuzzy ifthen rules, and fuzzy reasoning. It has found successful applications in a wide variety of fields, such as automatic control, data classification, decision analysis, expert systems, and pattern recognition [8]. The details of fuzzy logic can be found in [6].

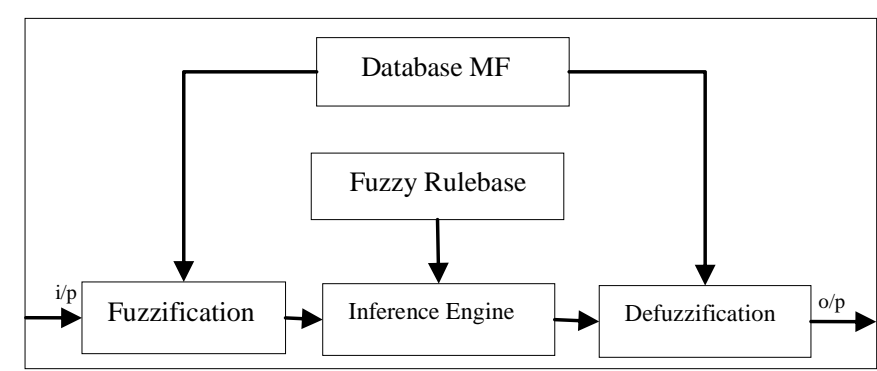

Fig 1: Framework of fuzzy logic system 


\subsection{Fuzzification}

The objective of the fuzzification is, to choose membership function, to transform the input from a numerical value into a set of fuzzy variables. The input is constantly a crisp numerical value restricted to the universe of discourse of the input variable and output is a fuzzy degree of membership in the qualifying linguistic set (always the interval 0 and 1 ). The membership function $\mu_{A}$ of a fuzzy set $\mathrm{A}$ is expressed as:

where $\chi$ is a universal set

$$
\mu_{A}: \chi \rightarrow[0,1]
$$

\subsection{Rule Based Reasoning}

Fuzzy sets are an aid in providing symbolic knowledge information in a more human understandable or natural form, and can hold uncertainties at various levels [15]. Manipulating a fuzzy rule based system involves the derivation of the desired 'If-Then' fuzzy rules, partitioning of universes, and addressing of the membership functions. The membership function used for distance measure classes are shown in "figure 2". Fuzzy rules can be easily and directly formulated by experts in the form of linguistic rules [7]. Fuzzy rule-based systems use linguistic variables to rationale using a series of logical rules that contain IF-THEN rules which connect antecedent(s) and consequent(s), respectively. An antecedent is a fuzzy clause with a certain degree of membership (between 0 and 1). Fuzzy rules can have multiple antecedents connected with AND or OR operators, where all parts are considered simultaneously and resolved into a single number. Consequents can also be comprised of multiple parts, which are then aggregated into a single output of a fuzzy set [2]. Accuracy based rule based systems offer a number of advantages, such as, it can distinguish between accurate and over general rules: an over general rule will have relatively low accuracy since payoff will vary according to the inputs covered by the rule. Indeed, it has been shown that accuracybased approach can lead to the evolution of optimally general rules. Additionally it can maintain a both consistently correct and consistently incorrect rule which allows learning of a complete "covering map."

The ANFIS combines both fuzzy logic principle and the concept of the neural networks. The ANFIS has advantages such as smoothness property from the fuzzy principle and adaptability property from the neural networks training structure [12]. Using advantageous of processing partial truth, it has been greatly utilized in engineering applications mainly in the form of FL controllers (FLC's), fuzzy models and fuzzy expert systems.

\subsection{Defuzzification}

Defuzzification is mapping process produces a non-fuzzy control action that best represents the possibility distribution of an inferred fuzzy control action. The defuzzification has the capability to reduce fuzzy set into a single-valued quantity or into a crisp set; to convert the fuzzy matrix into a crisp number. Various defuzzification methods include Maxmembership, centroid method, mean-max, the center of sums, the center of largest area etc. Although there is no unique defuzzification method and each defuzzification method has advantages and disadvantages, the centroid method is one of the most common approaches, which returns the center of the area under the curve. It can be expressed using Eq. (1)

$$
X_{d}^{T}=\frac{\int_{x} \mu_{c}^{\prime}(x) \cdot x d x}{\int_{x} \mu_{c}^{\prime}(x) d x}
$$

Where $\mu_{C}(x)$ is the membership function of output fuzzy set $\mathrm{C}^{\prime}$ obtained by the inference engine. The fuzzy rules can be expressed as, if $\mathrm{x}$ is small and $\mathrm{y}$ is middle, then $\mathrm{z}$ is larger distance covered. The variables $\mathrm{x}, \mathrm{y}$ and $\mathrm{z}$ are of linguistic type [14].

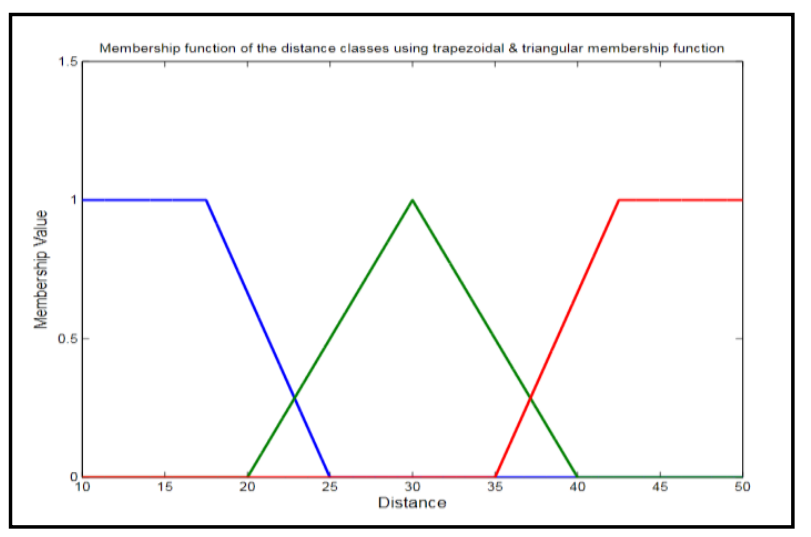

Fig 2: Membership function of the distance classes constructed using membership function

Fuzzy systems are fuzzy model structures in the form of fuzzy rule bases (FRBs) that are the most important area in the application of the fuzzy set theory [7] and have been successfully applied to many nonlinear control problems with the main advantages being they provide a framework to incorporate linguistic fuzzy information from human experts. Fuzzy inference system is operational with learning capability; all the design methodologies for neural network controllers become directly appropriate to fuzzy controllers [6]. Depending on the types of fuzzy reasoning and fuzzy ifthen rules employed, most fuzzy inference can be classified into two types, first one is Mamdani FIS proposed by Ebsahim Mamdani in 1975 to control the steam engine by synthesizing a set of fuzzy rules. The overall model output is expected to be fuzzy sets. Mamdani-type FIS is widely used in particular for decision support application. It includes linguistic models based on collections of IF-THEN rules, whose antecedents and consequents utilize fuzzy values. It uses fuzzy reasoning and the system behavior can be described in natural terms [15]. The maximum operator is used to compute the fuzzy output. The output Mamdani-type FIS uses the technique of defuzzification to compute fuzzy output [9]. Knowledge Rule-base can be described as:

$$
\begin{array}{r}
\mathrm{R}_{1}: \text { if } \mathrm{x}_{1} \text { is } A_{1}^{i} \text { and } \mathrm{x}_{2} \text { is } A_{2}^{i} \ldots \text { and } \mathrm{x}_{\mathrm{n}} \text { is } A_{m}^{i}, \\
\text { then } \mathrm{y}^{i} \text { is } \mathrm{B}^{\mathrm{i}} \ldots \ldots \ldots \ldots \ldots \ldots \ldots \ldots \ldots \ldots \ldots \ldots
\end{array}
$$

where, $R^{i}(i=1,2,3 \ldots 1)$ denotes $i^{\text {th }}$ fuzzy rule, $x_{j}(j=1,2,3 \ldots n)$ is the input, $\mathrm{y}^{\mathrm{i}}$ is the output of fuzzy rule, $\mathrm{R}^{\mathrm{i}}$ and $A_{i}^{1}, A_{1}^{2} \ldots A_{m}^{i}$, $\mathrm{B}^{\mathrm{i}}(\mathrm{i}=1,2 \ldots 1)$ are fuzzy membership function associated with linguistic terms.

Takagi-Sugeno fuzzy model (TS Method) was proposed by Takagi, Sugeno and Kang in year 1985 and can describe a wide class of nonlinear systems. Sugeno-type systems, uses a rule structure that has fuzzy antecedent and functional consequent parts. This approach approximates a nonlinear system with a combination of several linear systems, by decomposing the whole input space into several partial fuzzy spaces and the output of each rule is a linear arrangement of input variables plus a constant term [15]. A typical fuzzy rule in a Sugeno fuzzy model has the form: 


$$
\begin{gathered}
\mathrm{R}^{\mathrm{i}} \text { : if } \mathrm{x}_{1} \text { is } A_{1}^{i} \text { and } \mathrm{x}_{2} \text { is } A_{2}^{i} \ldots \text { and } \mathrm{x}_{\mathrm{n}} \text { is } A_{m}^{i} \text {, } \\
\text { then } \mathrm{y}^{\mathrm{i}}=\mathrm{a}_{0}+a_{1}^{i} \mathrm{x}_{1}+\ldots+a_{n}^{i} \mathrm{x}_{\mathrm{n}} \ldots \ldots \ldots \ldots \text { (3) }
\end{gathered}
$$

Usually yi represents a polynomial in the input variables. When yi is a first-order polynomial, the resulting fuzzy inference system is called a first-order Sugeno fuzzy model [6]. We use zero-order Sugeno fuzzy model for cases $f$ is constant. The overall output is obtained via weighted average, as each rule has a crisp output and thus excessive computational complexity in the procedure of defuzzification is avoided. This type of knowledge representation does not allow the output variables to be described in linguistic terms and the parameter optimization is carried out iteratively using a nonlinear optimization method [15]. Fuzzy rules, as produced by the data mining algorithm, model a behavior represented by the given data set. The output of the Fuzzy Inference Engine is the firing strength of each rule for a given fact, which determines whether or not the fact satisfy the modeled behavior [16]. Fuzzy logic widely used in many applications. In the area of control engineering, the fuzzy logic controller with many varieties and structures has been practically developed in the many areas such as control, forecasting, identification, and etc. The strongest thing of fuzzy logic's can adopt intuition of human feeling mapped in the membership function of fuzzy logic [17].

\section{ADAPTIVE NEURO-FUZZY INFERENCE SYSTEM}

This section describes a class of Neuro-Fuzzy along with the architectures and learning procedures of adaptive networks. The underlying network structure is a superset of all kinds of neural network paradigms with supervised learning capability. Neuro-fuzzy systems, is the combination of ANN with fuzzy systems, usually have the advantage of allowing an easy translation of the final system into a set of if-then rules, and the fuzzy system can be viewed as a neural network structure with knowledge distributed throughout connection strengths. Research and applications on neuro-fuzzy inference strategy made clear that neural and fuzzy hybrid systems are beneficial in fields such as the applicability of existing algorithms for artificial neural networks (ANNs), and direct adaptation of knowledge articulated as a set of fuzzy linguistic rules. An adaptive network, as its name implies, is a network structure consisting of nodes and directional links, overall input-output behavior is determined by the values of a collection of modifiable parameters through which the nodes are connected [6]. The adaptive system uses a hybrid learning algorithm to identify parameters of Sugeno-type fuzzy inference systems. It applies a combination of the least-squares method and the back-propagation gradient descent method for training FIS membership function parameters to emulate a given training data set [8].The network learns in two main phases. In the forward phase of the learning algorithm, consequent parameters identify the least squares estimate. In the backward phase, the error signals, which are the derivatives of the squared error with respect to each node output, propagate backward from the output layer to the input layer. In this backward pass, the premise parameters are updated by the gradient descent algorithm. Learning or training phase of the neural network is a process to determine parameter values to sufficiently fit the training data. ANFIS training can use alternative algorithms to reduce the error of the training. A combination of the gradient descent algorithm and a least squares algorithm is used for an effective search for the optimal parameters. The main benefit of such a hybrid approach is that it converges much faster, since it reduces the search space dimensions of the backpropagation method used in neural networks [2]. ANFIS are the fuzzy Sugeno model put in framework of the adaptive system which serves in model building and validation of developed model to facilitate training and adaptation [11].

\subsection{Architecture of ANFIS}

An adaptive network is a multilayer feed-forward network composed of nodes connected by directed links, in which each node performs a particular function on its incoming signals to generate a single node output. Each link in an adaptive network specifies the direction of signal flow from one node to another; no weights is associated with the link. More specifically, the configuration of an adaptive network performs a static node function on its incoming signals to generate a single node output and each node function is a parameterized function with modifiable parameters; by changing these parameters, the node functions as well as the overall behavior of the adaptive network, are changed. "Figure 3" shows entire system architecture consists of five layers, namely fuzzy layer, product layer, normalized layer, de-fuzzy layer and total output layer. With input/output data for given set of parameters, the ANFIS method models a fuzzy inference system (FIS) whose membership function parameters are tuned (adjusted) using either a backpropagation algorithm alone, or in permutation with a least squares type of method. The main objective of the ANFIS is to determine the optimum values of the equivalent fuzzy inference system parameters by applying a learning algorithm. The parameter optimization is done in such a way during the training session that the error between the target and the actual output is minimized. A hybrid algorithm is used for optimization, which is the combination of least square estimate and gradient descent method. The parameters to be optimized in ANFIS are the premise parameters. These parameters define the shape of the membership functions [18]. In order to reduce the error measure, any of several optimization routines can be applied after constituting MFs. The parameter set of an adaptive network allows fuzzy systems to learn from the data they are modeling. This paper assumes that adaptive system under consideration has two inputs V1 and V2 and one output $\mathrm{f}$. Let us scrutinize a first order Takagi, Sugeno and Kang (TSK) fuzzy inference system containing two rules:

Rule 1: If $\left(v\right.$ is $\left.V_{1}\right)$ and $\left(d\right.$ is $\left.D_{1}\right)$ then $f_{1}=p_{1} v+q_{1} d+r_{1}$

Rule 2: If $\left(v\right.$ is $\left.\mathrm{V}_{2}\right)$ and $\left(d\right.$ is $\left.\mathrm{D}_{2}\right)$ then $f_{2}=p_{2} v+q_{2} d+r_{2}$

Where $\mathrm{p}_{1}, \mathrm{p}_{2}, \mathrm{q}_{1}, \mathrm{q}_{2}, \mathrm{r}_{1}$ and $\mathrm{r}_{2}$ are linear parameters and $\mathrm{V}_{1}, \mathrm{~V}_{2}$, $D_{1}$ and $D_{2}$ are non linear parameters, in which $V_{1}$ and $D_{1}$ are the membership functions of ANFIS (antecedent). $\mathrm{p}_{1}, \mathrm{q}_{1}, \mathrm{r}_{1}$ are the consequent parameters [17]. To reflect adaptive capabilities, we use both circle and square. A circle indicates fixed node whereas square indicates adaptive node i.e. the parameter can be changed during adapting or training. ANFIS is created from integration of fuzzy logic and neural network.

While designing of ANFIS model, it is extremely important that the number of training epochs, the number of membership functions and the number of fuzzy rules should be tuned accurately. Mapping of those parameters is highly crucial for the system because it may lead system to over fit the data or will not be able to fit the data. This adjusting can be obtained by using a hybrid algorithm combining the least-squares method and the gradient descent method with a mean square error method. The lesser difference between ANFIS output and the desired objective means a better (more accurate) ANFIS system. So we tend to reduce the training error in the training process [13]. The integration between fuzzy logic and 
neural network namely fuzzy neural network (FNN) has been expected and developed; generally the arrangement of fuzzy logic and the neural network is called as ANFIS. Neural system has many inputs and also has multiple outputs, but the fuzzy logic has copious inputs and single output, so the combination of this two is known as ANFIS.

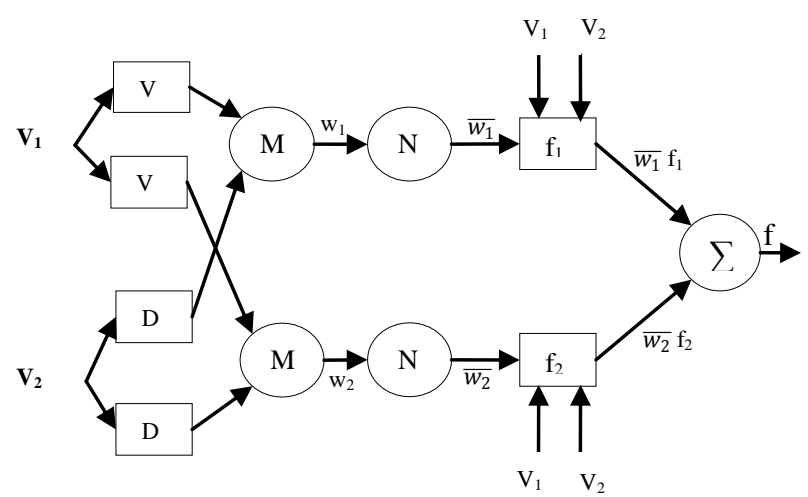

Fig 3: Basic architecture of ANFIS

\subsection{Layers of ANFIS}

For simplicity, the fuzzy inference system is under consideration of two inputs $v, d$ and one output $f$. A brief summary of five layers of the ANFIS algorithm is shown below.

\subsubsection{Layer 1}

Each input node $\mathrm{i}$ in this layer is an adaptive node which produce membership grade of linguistic label. It is a fuzzy layer, in which $\mathrm{v}$ and $\mathrm{d}$ are input of system. $\mathrm{O}_{1, \mathrm{i}}$ is the output of the $i^{\text {th }}$ node of layer 1 . Each adaptive node is a square node with square function represented using Eq. (4):

$$
\begin{aligned}
& O_{1, i}=\mu_{v, i}(v) \text { for } i=1,2 \\
& O_{1, j}=\mu_{d, j}(v) \text { for } j=1,2
\end{aligned}
$$

Where $\mathrm{O}_{1, \mathrm{i}}$ and $\mathrm{O}_{1, \mathrm{j}}$ denote output function and $\mu_{\mathrm{v}, \mathrm{i}}$ and $\mu_{\mathrm{d}, \mathrm{j}}$ denote membership function. For example if we choose triangular membership function, $\mu_{\mathrm{v}, \mathrm{i}}(\mathrm{v})$ is given by:

$$
\mu_{\mathrm{vi}}(\mathrm{v})=\max \left[\min \left(\frac{v-a_{i}}{b_{i}-a_{i}}, \frac{c_{i}-v}{c_{i}-b_{i}}\right), 0\right]
$$

Where $\left\{\mathrm{a}_{\mathrm{i}}, \mathrm{b}_{\mathrm{i}}, \mathrm{c}_{\mathrm{i}}\right\}$ are the parameter of triangular membership function. In other example, if we choose $\mu_{\mathrm{v}, \mathrm{i}}(\mathrm{v})$ to be bell shaped is given by:

$$
?(\mathbf{v})=\frac{1}{1+\left\{\left(\frac{v-c_{i}}{a_{i}}\right)^{2}\right\}^{b_{i}}}
$$

Where $\left\{a_{i}, b_{i}, c_{i}\right\}$ are the parameter set that changes shapes of M.F accordingly. Value of $a_{i}$ and $c_{i}$ that can be adjusted to vary the center and width of membership function and then $b_{i}$ is used to control slopes at crossover points of next membership function. Parameters in this layer are referred to as 'premise parameter'.

\subsubsection{Layer 2}

This layer checks weights of each membership function, it receives input values $v_{i}$ from first layer and acts as a membership function to represent fuzzy sets of respective input variables. Every node in this layer is fixed node labeled with $\mathrm{M}$ and output is calculated via product of all incoming signals. The output in this layer can be represented using Eq. 7:

$$
O_{2, i}=w_{i}=\mu_{v, i}(v) \cdot \mu_{D j}(d), i=1,2
$$

Which are the firing strengths of the rules. In general, any Tnorm operator that performs fuzzy AND can be used as a node function in this layer.

\subsubsection{Layer 3}

Every node in this layer is fixed marked with circle labeled with $\mathrm{N}$, indicating normalization to the firing strength from previous layer. This layer performs pre-condition matching of fuzzy rules, i.e. they compute activation level of each rule, the number of layers being equal to number of fuzzy rules. The $i^{\text {th }}$ node in this layer calculate ratio of $i^{\text {th }}$ rule's strength to the sum of all rules firing strength. The output of this layer can expressed as $\overline{w_{i}}$ using Eq. 8:

$$
\mathrm{O}_{3, \mathrm{i}}=\overline{w_{i}}=\frac{w_{\mathrm{i}}}{w_{1}+w_{2}}, \quad \mathrm{i}=1,2
$$

For convenience, outputs of this layer will be called as normalized firing strengths.

\subsubsection{Layer 4}

This layer provides output values y, resulting from the inference of rules. The resultant output is simply a product of normalized firing rule strength and first order polynomial. Weighted output of rule represented by node function as:

$$
\mathbf{O}_{4, \mathrm{i}}=\overline{w_{i}} \mathbf{f}_{\mathrm{i}}=\overline{w_{i}}\left(\mathrm{p}_{\mathrm{i}} \mathbf{v}+\mathrm{q}_{\mathrm{i}} \mathrm{d}+\mathrm{r}_{\mathrm{i}}\right), \quad \mathrm{i}=\mathbf{1}, \mathbf{2}
$$

Where $\mathrm{O}_{4, \mathrm{i}}$ represents layer 4 output. In this layer, $\mathrm{p}_{\mathrm{i}}, \mathrm{q}_{\mathrm{i}}$ and $\mathrm{r}_{\mathrm{i}}$ are linear parameter or consequent parameter.

\subsubsection{Layer 5}

This layer is called output layer which sums up all the inputs coming from layer 4 and transforms fuzzy classification results into crisp values. This layer consists of single fixed node labeled as ' $\sum$ '. This node computes summation of all incoming signals calculated using Eq. 10.

$$
\mathrm{O}_{5, \mathrm{i}}=\sum_{i} \overline{w_{i}} \boldsymbol{f}_{i}=\frac{\sum_{i} w_{i} f_{i}}{w_{1}+w_{2}}, \quad \mathrm{i}=1,2
$$

Thus, it is observed that when the values of premise parameter are fixed, the overall output of the adaptive network can be expressed as linear combination of a consequent parameter. Constructed network has exactly the same function as a Sugeno fuzzy model. Overall output of a system (z) can be expressed as in Eq. 11. It can be observed that ANFIS architecture consists of two adaptive layers, namely the first layer and the fourth layer. The three modifiable parameters $\left\{\mathrm{a}_{\mathrm{i}}, \mathrm{b}_{\mathrm{i}}, \mathrm{c}_{\mathrm{i}}\right\}$ are so-called premise parameter in first layer and in the fourth layer, there are also three modifiable parameters $\left\{\mathrm{p}_{\mathrm{i}}, \mathrm{q}_{\mathrm{i}}, \mathrm{r}_{\mathrm{i}}\right\}$ pertaining to the first order polynomial. These parameters are so-called consequent parameters [11].

$$
\begin{aligned}
\mathrm{z}= & \frac{w_{1}}{w_{1}+w_{2}} f_{1}+\frac{w_{2}}{w_{1}+w_{2}} f_{2}+\cdots+\frac{w_{n}}{w_{n-1}+w_{n}} f_{n} \\
\mathrm{z}= & w_{1}\left(p_{1} Q+q_{1} M+\cdots+m_{1} F+r_{1}\right) \\
& +\ldots+\left(\bar{w}_{n}\left(p_{n} Q+q_{n} M+\cdots+m_{n} F+r_{n}\right)\right.
\end{aligned}
$$

\subsection{Learning Algorithm of ANFIS}

Neuro-adaptive learning techniques endow with a method for the fuzzy modeling procedure to learn information about a data set. It computes the membership function parameters that best allow the associated fuzzy inference system to track the given input/output data. The parameters associated with the membership functions changes through the learning process [19]. In order to more efficiently cope with real world problems, the task of the learning algorithm for this architecture is to tune all the modifiable parameters, to formulate the ANFIS output match the training data. To 
improve the rate of convergence, the hybrid network can be trained by a hybrid learning algorithm combining least square method and gradient descent method can be used. The least squares method can be used to identify the optimal values of the consequent parameter on the layer 4 with premise parameter fixed. Gradient vector provides a measure of how well the fuzzy inference system is modeling the input/output data for a given set of parameters. When the gradient vector is obtained, any of several optimization routines can be applied in order to adjust the parameters to reduce some error measure [19]. When the premise parameters are not fixed, then the search space becomes larger and the convergence of the training becomes slower. The hybrid algorithm is composed of a forward pass (LSM) and a backward pass (GDM). Once the optimal consequent parameters are found, backward pass starts. In the backward pass, errors are propagated backward and the premise parameters corresponding to the fuzzy sets in the input domain updated by gradient descent method [11]. ANFIS uses a combination of least squares estimation and back-propagation for membership function parameter estimation. Two passes in the hybrid learning algorithm for ANFIS shown in table 1

Table 1: Passes of Hybrid learning algorithm

\begin{tabular}{|l|l|l|}
\hline & Forward pass & Backward pass \\
\hline $\begin{array}{l}\text { Premise } \\
\text { parameters }\end{array}$ & Fixed & $\begin{array}{l}\text { Gradient } \\
\text { descent }\end{array}$ \\
\hline $\begin{array}{l}\text { Consequent } \\
\text { parameters }\end{array}$ & Least square & Fixed \\
\hline Signals & Node outputs & Error signals \\
\hline
\end{tabular}

The output error is used to adapt the premise parameters by means of a standard back-propagation algorithm to minimize the mean square error function defined by Eq. (12). It has been proven that this hybrid algorithm highly efficient in training the ANFIS [1].

$\mathbf{E}(\theta)=\sum_{i=1}^{m}\left(\mathbf{z}_{i}-\boldsymbol{a}_{i}^{T} \theta\right)^{2}=e^{T} e=(z-\boldsymbol{A \theta})^{T}(z-\boldsymbol{A \theta}) \ldots \ldots . .(12)$ Where $\mathrm{e}=z-A \theta$ is the error vector produced by a specific choice of $\theta$. In Eq. (12) the squared error is minimized and is called the least squares estimator (LSE) [7]. Therefore, the hybrid learning algorithm can be applied directly. More specifically, the error signals proliferate backward and the premise parameters are updated by Gradient Descent (GD) and node outputs go forward until layer 3 and the consequent parameters are identified by the Least Squares (LS) method. This hybrid learning is structured as by defining, linear and nonlinear parameters are illustrious each iteration (epoch) of GD update the nonlinear parameters, LS follows to identify the linear parameters [19].

\section{CONCLUSION}

We have described the architecture of adaptive network based fuzzy inference systems (ANFIS) with type-1 and type-2 reasoning mechanisms. By employing hybrid learning algorithm, the proposed architecture can improve the quality of generated relevant fuzzy if-then rules obtained from human experts to describe the input-output behavior of a complex system. These characteristics are especially useful for modeling systems for which there is no analytical description available, but behaviors can be described by the human expert. However, when human expert is not available, nor is a linguistic description, the design process of the model can still set up intuitively reasonable initial membership functions and start the learning process to generate a set of fuzzy if-then rules to approximate a desired output. The adaptive network structure can have a number of variants of the model, which greatly relieves the burden of the human designer.

\section{REFERENCES}

[1] Jang R., "ANFIS :Adaptive-Network-Based Fuzzy Inference System," IEEE Transactions on Systems, MAN, and Cybernetics, Vol. 23, No. 3, 1993.

[2] Hamdan H., Garibaldi M., "Adaptive Neuro-Fuzzy Inference System (ANFIS) in Modelling Breast Cancer Survival," WCCI 2010, IEEE World Congress on Computational Intelligence, pp. 18-23, 2010.

[3] Prasath V., Lakshmi N., Nathiya M., Bharathan N., Neetha P., "A Survey on the Applications of Fuzzy Logic in Medical Diagnosis Support Systems Systems Decision," International Journal of Scientific \& Engineering Research, ISSN 2229-5518, Vol. 4, No. 4, pp. 1199-1203, 2013.

[4] Qiu M., "Data Mining Based on Compensation Fuzzy Neural Networks," International Conference on Education, Management, Commerce and Society, International Conference on Education, Management, Commerce and Society, pp. 306-311, 2015.

[5] Mousavi J., Ponnambalam K., Karray F., "Inferring operating rules for reservoir operations using fuzzy regression and ANFIS," Fuzzy Sets and Systems, Elsevier, vol. 158, pp. 1064-1082, 2007.

[6] Jang R., "Neuro-Fuzzy Modeling," proceedings of the IEEE, vol. 83, no. 3, 1995.

[7] Taylan O., "An adaptive neuro-fuzzy model for prediction of student's academic performance," Computers \& Industrial Engineering, Elsevier, vol. 57, pp. 732-741, 2009.

[8] Rezaei K., Hosseini R., Mazinani M., “A Fuzzy Inference System for Assessment of the Severity of the peptic ulcers," Computer Science \& Information Technology, DOI:10.5121/ csit.2014.4527, pp. 263-271, 2014.

[9] Kaur A., Kaur A., "Comparison of Mamdani-Type and Sugeno-Type Fuzzy Inference Systems for Air Conditioning System," International Journal of Soft Computing and Engineering, ISSN: 2231-2307, no. 2, pp. 323-325, 2012.

[10] Efosa C., Akwukwuma N., "Knowledge based Fuzzy Inference System for Sepsis Diagnosis," International Journal of Computational Science and Information Technology, Vol.1, No. 3, pp. 1-7, 2013.

[11] Roy S., "Design of adaptive neuro-fuzzy inference system for predicting surface roughness in turning operation," Journal of Scientific \& Industrial Research, vol. 64, no. September, pp. 653-659, 2005.

[12] Choi H., Yoo H., Jung H., Lim T., Lee K., Ahn K., “An ANFIS-based Energy Management Inference Algorithm with Scheduling Technique for Legacy Device," International Conference on Artificial Intelligence, Energy and Manufacturing Engineering, pp. 80-82, 2015.

[13] Uc T., Karahoca A., Karahoca D., "Tuberculosis disease diagnosis by using adaptive neuro-fuzzy inference system and rough sets," Neural Comput \& Applications, Springer, DOI 10.1007/s00521-012-0942-1, pp. 471-483, 2013. 
[14] Neshat M., Adeli A., "A Comparative Study on ANFIS and Fuzzy Expert System Models for Concrete Mix Design," International Journal of Computer Science Issues, ISSN 1694-0814, Vol. 8, No. 3, pp. 196-210, 2011.

[15] S. Mitra, Y. Hayashi, S. Member, "Neuro Fuzzy Rule Generation: Survey in," IEEE transactions on neural networks, Vol. 11, No. 3, pp. 748-768, 2000.

[16] Semary A., Edmonds J., "A Framework for Hybrid Fuzzy Logic Intrusion Detection Systems," IEEE International Conference on Fuzzy Systems, pp. 325330, 2005.

[17] Pratama M., Rajab S., Joo E., "Extended Approach of ANFIS in Cascade Control," International Journal of Computer and Electrical Engineering, Vol. 3, No. 4, pp. $1-5,2011$.

[18] Patel D., Parekh F., "Flood Forecasting using Adaptive Neuro-Fuzzy Inference System," International Journal of Engineering Trends and Technology, ISSN: 2231-5381, vol. 12, no. 10, pp. 510-514, 2014.

[19] Power E., “Adaptive Neuro Fuzzy Inference System (ANFIS) For Fault Classification in the Transmission Lines," Online Journal on Electronics and Electrical Engineering, Ref: W09-0030, no. 2, pp. 164-169.

[20] Jhin C., Hwang T., "Prediction of Radical Scavenging Activities of Anthocyanins Applying Adaptive NeuroFuzzy Inference System(ANFIS) with Quantum Chemical Descriptors," International Journal of Molecular Science, DOI: 10.3390/ijms150814715, pp. 14715-14727, 2014.

[21] Gite A., Bodade M., Raut M., "ANFIS Controller and its Application," International Journal of Engineering Research \& Technology, Vol. 2, No. 2, pp. 1-5, 2013.

[22] Abu-rub H., Iqbal A., Ahmed M., Peng Z., Li Y., Baoming G., "Quasi-Z-Source Inverter-Based Photovoltaic Generation System With Maximum Power
Tracking Control Using ANFIS," IEEE Transactions on Sustainable Energy, Vol. 4, No. 1, pp. 11-20, 2013.

[23] Loganathan C., Girija V, "Investigations on Hybrid Learning in ANFIS," International Journal of Engineering Research \& Applications, Vol. 4, No. 10, pp. 31-37, 2014.

[24] Toosi N., Kahani M., "A Novel Soft Computing Model Using Adaptive Neuro-Fuzzy Inference System for Intrusion Detection," IEEE International Conference on Networking, Sensing and Control, pp. 15-17, 2007.

[25] Lavanya K., Durai S., Sriman C., Iyengar N., "Fuzzy Rule Based Inference System for Detection and Diagnosis of Lung Cancer," International Journal of Latest Trends in Computing, Vol. 2, No. 1, pp. 165-171, 2011.

[26] Guillaume S., "Designing Fuzzy Inference Systems from Data an Interpretability-Oriented Review," IEEE transactions on fuzzy systems, Vol. 9, No. 3, pp. 426443, 2001.

[27] Yardimci A., "Soft computing in medicine," Applied Soft Computing, Elsevier, vol. 9, pp. 1029-1043, 2009.

[28] Walia N., Tiwari K., Malhotra R., "Design and Identification of Tuberculosis using Fuzzy Based Decision Support System," Advances in Computer Science and Information Technology, ISSN: 2393-9915, vol. 2, no. 8, pp. 57-62, 2015.

[29] Kwong C., Chuah C., Lee W., "Adaptive Network Fuzzy Inference System Handoff Algorithm," International Journal of Network and Mobile Technologies, ISSN 1832-6758, vol. 1, no. 2, pp. 54-59, 2010.

[30] Obi C., A. Imainvan A., "Decision Support System for the Intelligient Identification of Alzheimer using Neuro Fuzzy logic," International Journal on Soft Computing, DOI : 10.5121/ijsc.2011.2203, vol. 2, no. 2, pp. 25-38, 2011. 